\title{
On Concurrent Multipath Transfer in SCTP-based handover scenarios
}

\author{
Łukasz Budzisz* Ramon Ferrús*, Ferran Casadevall* and Paul Amer ${ }^{\dagger}$
}

* Radio Communication Group (GCR),

Signal Theory and Communication Dept (TSC),

Universitat Politècnica de Catalunya (UPC), Spain

Email: [lukasz,ferrus,ferranc]@tsc.upc.edu

\author{
$\dagger$ Protocol Engineering Lab (PEL), \\ Computer and Information Sciences Dept (CIS), \\ University of Delaware, USA \\ Email: amer@cis.udel.edu
}

\begin{abstract}
Handling mobility at the transport layer is a promising approach to achieve seamless handover in the context of heterogeneous wireless access networks. In particular, features such as multihoming and dynamic address reconfiguration provided by mobile SCTP (mSCTP) protocol are among the key enablers for handover support at the transport layer. This paper investigates the applicability of Concurrent Multipath Transfer (CMT) to distribute data among two end-to-end paths of an mSCTP association during the handover transition process. To that end, the principles of the MSCTP-CMT design are given, emphasizing the consequences of a sender-introduced reordering and its effect on congestion control. The proposed mSCTPCMT handover scheme is benchmarked with two other handover schemes, namely mSCTP and SCTP failover-based. Provided analysis indicates the possible application area of mSCTP-CMT, taking into account not only handover scenario parameters (dwelling time, available bandwidth ratio and round-trip time), but also an important design constraint: receiver buffer (rbuf) size. Rbuf size proves to be a major limiting factor shrinking significantly, yet not excluding mSCTP-CMT's application scope.
\end{abstract}

\section{INTRODUCTION}

The Stream Control Transport Protocol (SCTP), defined in RFC 4960 [1] and further referred to as standard SCTP, provides transport-layer multihoming. Multihoming binds multiple source-destination IP addresses for a single association between two SCTP endpoints. These IP addresses are exchanged and verified during the association initiation, and define alternate paths between the corresponding peers. Multiple paths are distinguished at each endpoint by their destination addresses. Among all available destinations, one is selected as the primary destination, and the others are used as backup destinations. Multihoming, in the case of IP networks, means multiple IP addresses, and typically (but not necessarily) multiple link-layer interfaces.

Multihoming in SCTP was designed for environments requiring high application availability, such as the delivery of Signaling System No. 7 (SS7) messages. Hence its scope of use, defined within RFC 4960, is only for handling single retransmissions and performing a primary path failover in case of permanent link failure. Any other applications of multihoming, e.g., transport-layer handover or loadsharing are not supported by the standard SCTP specification, and instead should be subject of dedicated protocol extensions. This is the case with transport layer handover that reuses the Dynamic Address Reconfiguration (DAR) SCTP extension [2], whereas loadsharing is introduced in several different proposals (yet non-standardized by IETF) with the most common scheme called Concurrent Multipath Transfer (CMT) [3].

SCTP has migrated from the signaling environment to become an IETF general purpose transport protocol. SCTP's multihoming feature is attractive to wireless scenarios, capable of providing handover management at the transport layer. To accomplish the dynamic nature of handover, SCTP needed a mobility enabler, provided by the DAR extension [2]. Although originally defined to help with IPv6 renumbering and hot-pluggable cards, the DAR extension can be leveraged to make SCTP a mobility-enabled transport protocol [4]. The DAR extension allows either of the two SCTP endpoints to (1) dynamically add or delete IP addresses to an existing end-toend SCTP association, and (2) request the primary destination be changed during an active SCTP association. DAR defines two new chunk types ${ }^{1}$ : the Address Configuration Change (ASCONF) and the Address Configuration Ack (ASCONFACK); and six new parameters: Add IP Address, Delete IP Address, Set Primary Address, Error Cause Indication, Success Indication, Adaptation Layer Indication. Dynamically modifying IP address(es) of an association introduces a risk of association hijacking; therefore the ASCONF chunk must be authenticated (an authentication chunk is bundled before the ASCONF chunk), as described in [5]. Standard SCTP enhanced with the DAR extension is referred to as mobile SCTP (mSCTP) [4], [6].

Several studies have evaluated the performance of the mSCTP in the context of heterogeneous wireless networks, so called fourth-generation (4G) mobile data networks. The first analyses investigated the feasibility of mSCTP providing handover support at the transport layer. Authors concluded that mSCTP can supply seamless handover support [7], but still with important shortcomings, even after necessary protocol parameter adjustments [8]. Possibly the main deficiency lies in providing an appropriate handover policy (i.e., triggering

\footnotetext{
${ }^{1} \mathrm{~A}$ chunk is a unit of information within an SCTP packet.
} 


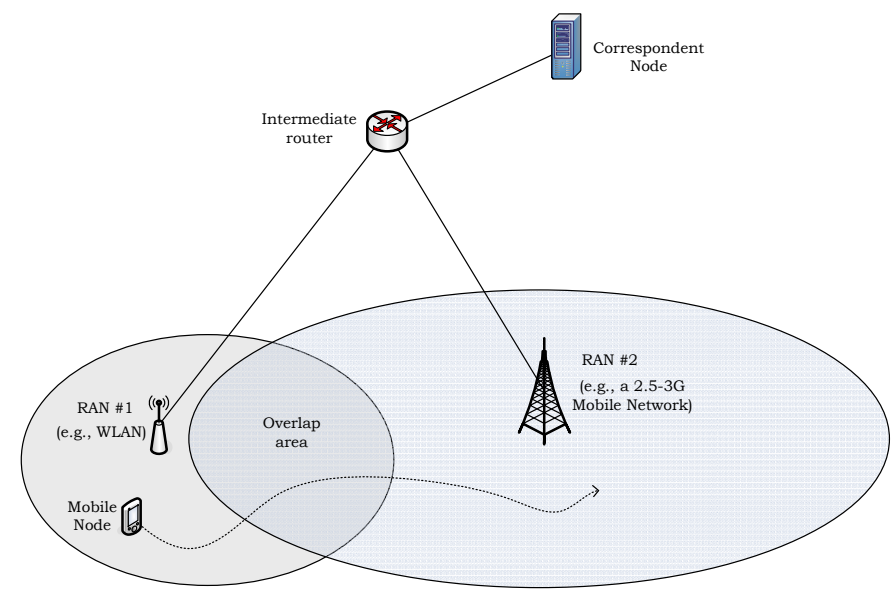

Fig. 1. Proposed CMT scenario.

condition for the primary destination change); its design can be approached also in a cross-layer fashion [9]. Budzisz et al. [10] survey mSCTP for transport-layer handovers, and conclude that not only the handover policy but also the improvements introduced to the transition process contribute to override mSCTP inadequacies. To that end, in this paper we provide the details of the CMT design and evaluate its applicability, both analytically and by means of simulations, as a potential enhancement to the handover schemes based on mSCTP that may smooth the transition process, and moreover, even improve the application's overall throughput.

The rest of the paper is organized as follows: Section II provides initial assumptions and description of the evaluating approach, whereas Section III explains insights of CMT's algorithms. Theoretical analysis, simulation results and discussion are provided in Section IV, followed by conclusions in Section V.

\section{SCENARIO AND ASSUMPTIONS}

A general handover scenario in heterogeneous wireless networks is shown in Fig. 1, where a mobile node (MN) is traversing one particular radio access network (RAN \#1) coverage area towards the coverage area of a neighboring RAN \#2. The RANs have an overlap area, i.e., an area where both RANs provide coverage. It is assumed that the $\mathrm{MN}$ is capable of handling transmissions on multiple links simultaneously. This assumption is fairly reasonable, as in the near future, nearly all mobile multimedia devices will be equipped with multiple network interfaces, despite the current power consumption constraints. Consequently, once an $\mathrm{MN}$ enters the overlap area, multiple links are physically available for simultaneous data transmission. This research examines the potential gain that can be achieved using CMT as part of the handover scheme in the overlap area.

It is further assumed the different paths do not share bottlenecks, i.e., the radio link of each path is the bottleneck. This is a coherent supposition taking into account the mixed wired-wireless topology of the envisaged scenarios, and as it

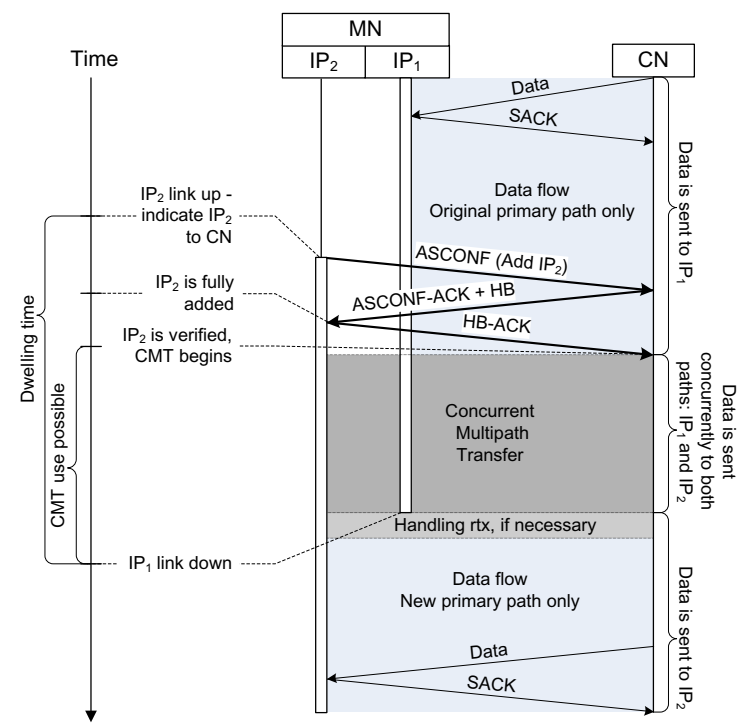

Fig. 2. Handover scheme with CMT.

will be explained later, it is vitally important for the use of CMT. As a consequence, the bottlenecks on each path are independent and a sender can consider a per-path congestion approach, while still preserving overall TCP-friendliness.

Now focusing on the handover scheme, this work considers one directional bulk data flow from a correspondent node (CN) to the MN. As presented in Fig. 2, a MN configured initially with $\mathrm{IP}_{1}$ address before entering an overlap area is using the mSCTP protocol to transfer data on a single link. When the MN enters the overlap area, the coverage of RAN \#2 is discovered. To get the new link operational, the MN undergoes the correspondent network registration procedure. Both the network discovery process and registration procedure details [11] are outside the scope of this work. As soon as the network address $\mathrm{IP}_{2}$ in RAN \#2 is operational, the CN must be informed about the new destination (by means of ASCONF chunk), and has to verify its availability (sending HEARTBEAT (HB) chunk). Once the new destination is confirmed, the $\mathrm{IP}_{2}$ address is considered available for normal data transfer. At this point, CMT can be exploited while having two paths available ${ }^{2}$. When the MN leaves the overlap area, it is necessary to: (1) quit CMT mode, (2) handle any retransmissions of packets that were in flight on the link that just went down, and (3) perform all necessary congestion adjustments on the current path for the once again singlehomed MN.

Our main goal is to specify how to apply CMT in Fig. 1's handover scenario, what gains can be achieved, and in which situations, if any, might CMT degrade service. To this end, the described CMT handover scheme (Fig. 2) will be compared

\footnotetext{
${ }^{2}$ Implementation details of CMT are described in Section III.
} 


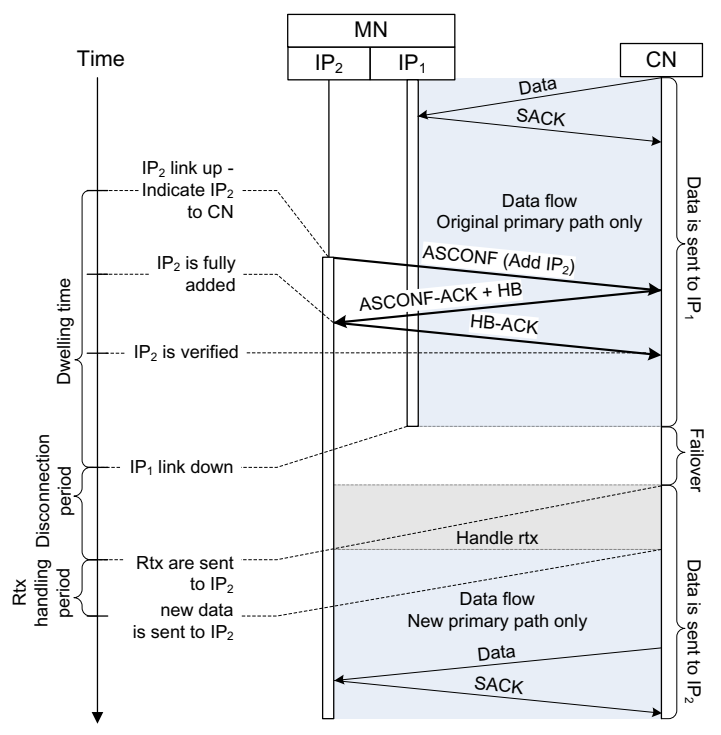

Fig. 3. Handover scheme for optimized standard SCTP with failover as a triggering mechanism for handover.

to two benchmark schemes:

- a handover based on the optimized failover mechanism of standard SCTP, as illustrated in Fig. 3. To optimize for handover scenarios, standard SCTP's failover mechanism was tailored with low Path.Max.Retrans (PMR) settings and the $R T O_{\min }$ limitation was removed, as described in [10]. Note that the usage of SCTP's failover mechanism to trigger primary path change impedes sending data while switching the paths.

- a handover based on mSCTP, as illustrated in Fig. 4. As mentioned in Section I, an important challenge for $\mathrm{mSCTP}$ is to provide an appropriate handover policy, i.e., to optimally select the instant when the ASCONF chunk with Set Primary parameter should be sent. Therefore, it may be considered beneficial for some handover policies to bundle a Set Primary with Add/Delete IP Address parameter within one ASCONF chunk. To give the reader more insight on this issue, two ideal schemes reflecting a range of possible adjustments to the envisaged scenario are provided: (1) the best case, a policy resulting in $\mathrm{MN}$ staying in the better quality RAN (in terms of bandwidth, propagation delay or both) as long as possible, and (2) a worst case keeping the $\mathrm{MN}$ in the poorer quality RAN for the maximum duration.

Lastly, we introduce two important parameters to evaluate CMT performance:

1) dwelling time $\left(t_{d w e l l}\right)$, defined as the effective time a MN remains in the overlap area. Dwelling time is affected by the speed of the $\mathrm{MN}$, as well as its movement pattern, and therefore may be crucial for using CMT. Depending on the scenario considered, $t_{d w e l l}$ can vary

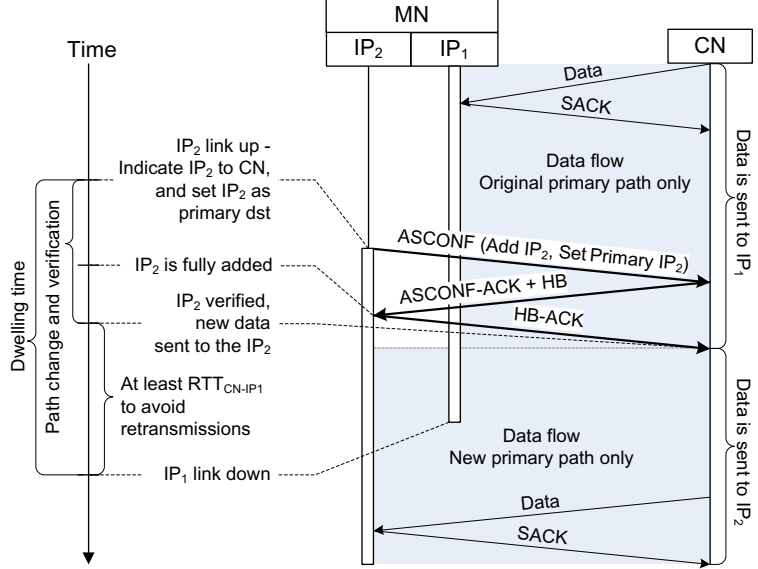

Fig. 4. Handover scheme for mSCTP with handover policy triggering the path change, as soon as the new path is discovered.

in practice from a few seconds for fast MNs going across the overlap area to tenths of seconds for slow MNs traversing the overlap area.

2) bandwidth ratio ( $\left.b w_{\text {ratio }}\right)$, defined as a ratio of the bandwidths available in the neighboring RANs (in this work the faster bandwidth is related to the slower one, so the $\left.b w_{\text {ratio }} \geq 1\right)$. bw $w_{\text {atio }}$ reflects the asymmetry of a handoff scenario.

\section{CMT SCHEMES}

To introduce CMT into a mSCTP-based handover scheme, a few important aspects must be considered. First, the standard SCTP's congestion control algorithms must be updated to take into account the problems of sending data over multiple paths using the single sequence number space. The consequences of sender-introduced reordering are described in Section III-A. Second, in wireless scenarios, the fixed Internet's basic assumption that losses are due to congestion is invalid. Wireless links are more prone to packet corruption than to congestion problems. Therefore, it is reasonable to expect failures more often in wireless scenarios, and to reduce their impact by applying the CMT's Potentially Failed (CMT-PF) solution proposed in [12] that will be described in Section III-B. The CMT scheme under test described in Section IV will comprise both modifications.

\section{A. Congestion control modifications}

Accomplishing CMT for SCTP implies a new sender architecture, where each path (not necessarily interface) must have a separate buffer to guarantee path independence. This modification preserves TCP-friendliness under the assumption that no bottleneck is shared by the multiple paths, which is the case in the analyzed scenario (a mixed wired-wireless topology with single hop wireless part). Of course, such a multi-buffer sender structure has its implications on congestion 
control, and therefore several changes must be considered. All algorithms cited here and incorporated to wireless scenarios in our analysis, were proposed and tested in fixed networks by Iyengar et al. [3]:

1) congestion control is handled per-path, not per association, so it is possible to have different congestion window (cwnd) evolution schemes, as functions of the conditions on each path. Thus, Selective Acks (SACKs) updating the Cumulative Transmission Sequence Number ACK Point (CumTSN) received in-order per path and out-of-order per association should increase the cwnd on that path. To track the earliest outstanding TSN per destination, a sender cwnd growth algorithm (cwnd update for CMT - CUC) has been proposed.

2) fast retransmission needs slight modification as reordering introduced on the sender side can provoke unnecessary spurious fast retransmissions with cwnd implications. Elimination of spurious fast retransmissions is handled by the Split Fast Retransmit (SFR) algorithm.

3) a data receiver should not send immediate SACKs on the arrival of duplicate packets as networks may be vulnerable for the increased ACK traffic. Therefore an algorithm Delayed ACK for CMT (DAC) was applied.

4) appropriate retransmission policy for handling retransmissions. Out of five different policies proposed by Iyengar in [3], the best results for bulk transfer were achieved by the loss rate-based policies. One of them, the RtxCwnd policy (retransmit on the path with the highest cwnd), is used in our experiments.

In addition, CMT can provoke the following problems that must be taken into account when considering handover scenarios:

1) receiver buffer blocking (receiver buffer is filled with out-of-order data) caused by complete or short-term failures. This problem is partially mitigated by the CMTPF solution described in Section III-B.

2) an ambiguity at the sender for the SACKs with the same CumTSN that ack various Gap ACK blocks: first more Gap ACK blocks are acked on the faster path followed then by a packet with fewer Gap ACK blocks received on the slower path. This can lead to an unnecessary retransmission in case the difference between paths' bandwidth is high.

3) incorrect round-trip time (RTT) estimate on a slower path that comes from the ambiguity of the SACK received on the faster path that also acks the TSN marked for a RTT estimation on the slower path.

Above problems will be referred to, when commenting the analysis results.

\section{B. CMT-PF extension}

To reduce the receiver buffer problem in CMT, the Potentially Failed (PF) solution was proposed in [12]. A path that experiences a single timeout is marked as a "potentially failed" and no further data transmission is allowed on that path.
Instead, a HB packet is sent every RTO to probe the path, and either the path gets back to an active state in case of successful HB packet transmission, or the path is considered inactive as if the PMR threshold were exceeded (with PMR +1 consecutive failures). The PF state prevents the PMR parameter settings from degrading the throughput performance during failure scenarios, as the exponential backoff mechanism clocks only the HB packets.

\section{Performance eVAluation}

Our proposed scenario under test (Fig. 1) considers the following mobility pattern: (1) first the MN moves within the coverage area of RAN \#1 (faster of the two), (2) after $t_{1}$ from the transmission start, the MN enters the overlap area where there is a possibility of applying CMT scheme during $t_{d w e l l}$, and finally (3) MN leaves the overlap area and remains in RAN \#2 (slower RAN), where again only one path is available for data transmission. Before looking for the simulation results of the CMT in such a defined handover scenario, we estimate the maximum possible gain that can be achieved, and relate it to mSCTP-based handover schemes.

\section{A. Analytical model}

According to the mobility pattern described for the analyzed scenario, the minimum time necessary to transmit file of size $L$ (given that $\mathrm{L}$ is large enough not to complete the transmission before leaving the overlap area) depends on the available bandwidth that the MN can achieve in each of the discussed regions. Hence:

$$
T=\sum_{i=1}^{3} t_{i}=\sum_{i=1}^{3} \frac{L_{i}}{b w_{i}}=\frac{L_{1}}{b w_{1}}+\frac{L_{\text {overlap }}}{b w_{\text {overlap }}}+\frac{L_{2}}{b w_{2}}
$$

As can be easily seen from formula (1) the main factor differentiating the performance of all discussed handover schemes is the bandwidth available in the overlap area $\left(b w_{\text {overlap }}\right)$. If CMT is applied in the analyzed scenario with two paths available for using CMT during the $t_{d w e l l}$, where $a_{1}$ and $a_{2}$ are the corresponding current available bandwidths, the bworlap can be estimated as:

$$
\text { bwoverlap }=a_{1}+a_{2}
$$

The maximum gain will be produced when the $a_{1}$ and $a_{2}$ would be equal to the values of link bandwidth, $b w_{1}$ and $b w_{2}$. Therefore, theoretically the minimum time necessary to transmit the entire file of size $L$ when CMT is applied in the overlap area is:

$$
T_{c m t_{-} t h}=t_{1}+t_{d w e l l}+\frac{L-t_{1} \cdot b w_{1}-t_{d w e l l} \cdot\left(b w_{1}+b w_{2}\right)}{b w_{2}}
$$

leading to:

$$
T_{c m t_{-} t h}=t_{1}+\frac{L}{b w_{2}}-\left(t_{1}+t_{d w e l l}\right) \cdot b w_{\text {ratio }}
$$


For mSCTP-based handover schemes the handover policy will influence the value of bwoverlap, changing its value from $\min \left(b w_{1}, b w_{2}\right)$ (bandwidth of the slower of the two RANs) in the worst case to $\max \left(b w_{1}, b w_{2}\right)$ in the best case, during the time the MN stays in the overlap area $\left(t_{d w e l l}\right)$. Therefore, corresponding file transfer times are,

for the mSCTP worst case:

$$
T_{m s c t p_{-} w o r s t}=t_{1}+\frac{L}{b w_{2}}-t_{1} \cdot b w_{\text {ratio }}
$$

and for the mSCTP best case:

$$
T_{m s c t p \_b e s t}=t_{1}+t_{d w e l l}+\frac{L}{b w_{2}}-\left(t_{1}+t_{d w e l l}\right) \cdot b w_{\text {ratio }}
$$

Finally, the maximum theoretical gain of mSCTP-CMT over mSCTP schemes $(\Delta T)$ can be expressed as:

$$
\begin{aligned}
& \Delta T \in {\left[\Delta_{\text {min }}, \Delta_{\text {max }}\right]=} \\
& {\left[T_{\text {msctp_best }}-T_{\text {cmt_th }}, T_{\text {msctp_worst }}-T_{\text {cmt_th }}\right] } \\
& \Delta T \in\left[t_{\text {dwell }}, t_{\text {dwell }} \cdot b w_{\text {ratio }}\right]
\end{aligned}
$$

Moreover, equation (3) expressing the best theoretical time for the handover scheme with CMT will be used to benchmark the results obtained in the simulations.

\section{B. Simulations results}

To study the feasibility of using CMT in the SCTP handover, simulation experiments in ns-2 (ver. 2.32) [13] were conducted, adjusting an existing CMT-PF implementation to work in a heterogeneous wireless environment. The important simulation parameters of the scenario under test are presented

\begin{tabular}{|c|c|}
\hline PARAMETER NAME & VALUE / RANGE \\
\hline Wired part (each path) & $\begin{array}{r}\text { bandwidth: } 100 \mathrm{Mbps} \\
\text { propagation delay: } 5 \mathrm{~ms}\end{array}$ \\
\hline RAN \#1 (fast RAN) & $\begin{array}{r}\text { bandwidth: }\left(\text { bw } w_{\text {ratio }} \times 384\right) \mathrm{kbps} \\
\text { propagation delay: } 15 \mathrm{~ms}\end{array}$ \\
\hline RAN \#2 (slow RAN) & $\begin{array}{r}\text { bandwidth: } 384 \mathrm{kbps} \\
\text { propagation delay: } 80 \mathrm{~ms}\end{array}$ \\
\hline bw $w_{\text {ratio }}$ & $1-14$ \\
\hline$t_{\text {dwell }}$ & $2-40 \mathrm{~s}$ \\
\hline rbuf size & $\begin{array}{r}16-256 \mathrm{kB} \\
\text { (ideal buffer up to } 2 \mathrm{MB} \text { ) }\end{array}$ \\
\hline RTOMin & $50 \mathrm{~ms}$ \\
\hline PMR & $\begin{array}{r}\text { Optimized failover, mSCTP: } 1 \\
\text { CMT: } 5\end{array}$ \\
\hline SACK delay & $200 \mathrm{~ms}$ \\
\hline Retransmission policies & $\begin{array}{l}\text { mSCTP FastRtx: Same path } \\
\text { mSCTP TimeoutRtx: Alternate path } \\
\text { CMTRtx: path with largest cwnd }\end{array}$ \\
\hline MTU size / Data payload & $1500 / 1468$ Bytes \\
\hline File size & $8 \mathrm{MB}$ \\
\hline
\end{tabular}
in Table I. Note that proposed RANs settings correspond to WLAN for RAN \#1, and UMTS for RAN \#2, respectively.

TABLE I

SIMULATION PARAMETERS



Fig. 5. Performance comparison of all SCTP versions for $b w_{\text {ratio }}=4$, and $t_{d w e l l}=40 \mathrm{~s}$.

First, in Fig. 5 a performance comparison in terms of TSNtime evolution diagram is presented for all SCTP protocol versions described in Section II, namely: (1) optimized failover, (2) mSCTP-based solution (both best and worst case), and (3) the mSCTP-CMT scheme (which includes CMT-PF, but herein is referred to as CMT). Additionally, obtained results are related to a single link SCTP performance (faster of the two available). As can be seen in Fig. 5, the overlap area $(2-42 \mathrm{~s}$ in the time scale) is the zone of special interest, witnessing different slope values for presented SCTP flavors. Any possible CMT gain over existing handover schemes will occur here, if strict constraints on $t_{d w e l l}$, as well as rbuf size are met. The range for possible CMT performance gain is significant, filling the area between the worst mSCTP policy and beyond the faster of the two links (for an appropriate CMT adjustment). Therefore it is essential that CMT fits the scenario to which is applied, otherwise reordering introduced at the sender heavily deteriorates overall performance. In an effort to identify the application area for CMT, all important factors such as, $t_{d w e l l}, b w_{\text {ratio }}$ and rbuf size are analyzed now.

As seen in dwelling time graph (Fig. 6), users with short $t_{d w e l l}$ would prefer choosing the appropriate moment to switch the interfaces rather than use CMT, as no significant gain can be produced when compared to mSCTP-based schemes. In contrast, having a long $t_{d w e l l}$ can effectively benefit from CMT. This conclusion follows the results of theoretical analysis, and so do the respective gains of CMT over both extreme cases of mSCTP. However, again the impact of rbuf blocking can be witnessed. Within our tested scenario CMT was not capable of outperforming best $\mathrm{mSCTP}$ case for a rbuf not exceeding $256 \mathrm{kB}$. Having an ideal rbuf (i.e., large enough to avoid rbuf blocking) led to nearly optimal performance marked by the theoretical trend based upon equation (3).

To better understand the receiver buffer blocking problem, in Fig. 7, the following metrics are provided: the smallest rbuf 


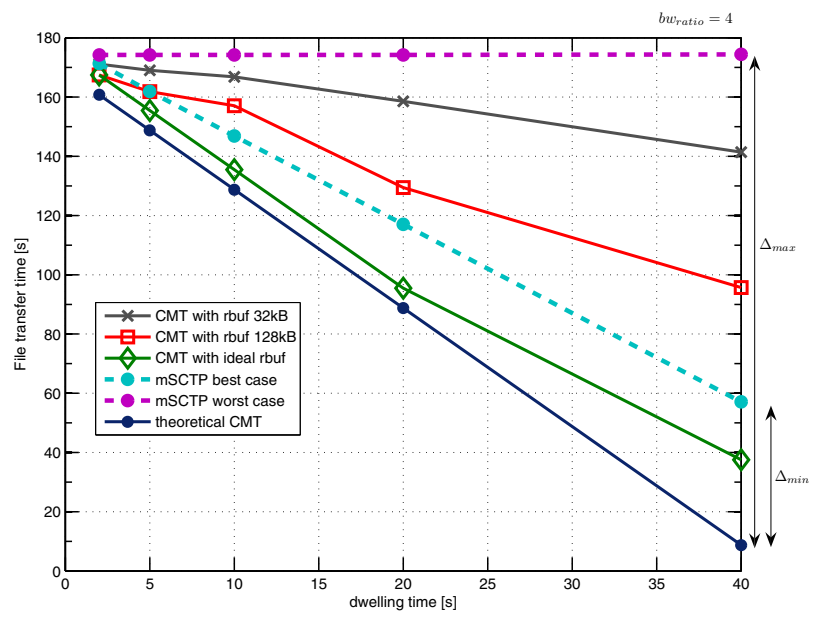

Fig. 6. Comparison in function of $t_{d w e l l}$ for bwatio $=4$.

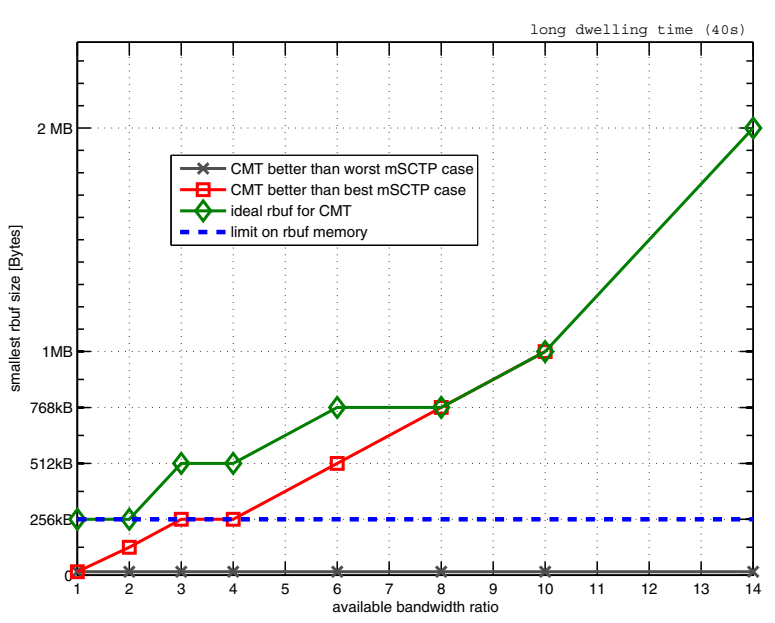

Fig. 7. Rbuf size constraints.

size that guarantees CMT outperforms mSCTP in terms of overall file transfer, for worst and best policy accordingly, as well as the smallest rbuf size without the rbuf blocking problem at all. The result is clear, not much asymmetry between two paths is allowed. Assuming a 256kB limitation on rbuf memory at $\mathrm{MN}$ is reasonable nowadays, only a $b w_{\text {ratio }} \leq 2$ makes the CMT application feasible, if the design concern is not having rbuf blocking at all. Less conservatively, if improvement over the mSCTP best case is the sole design goal, CMT scope of use extends to $b w_{\text {ratio }} \leq 4$, a value that would correspond, for instance, to a handover from WLAN to UMTS. Beyond this limit, the difference between both links makes the application of CMT pointless.

\section{CONCLUSION AND FURTHER WORK}

In this paper we proposed and analyzed the applicability of CMT (or strictly speaking CMT-PF) to the mSCTP-based transport-layer handover scenarios, as having the potential of improving the handover process between two paths. Provided that mSCTP itself lacks handover policies, CMT can be seen as an added value to such mSCTP scheme. An initial evaluation demonstrates a strong influence of receiver buffer blocking on any scenario where CMT is used, resulting in firm limitation of possible application area in terms of receiver buffer size. The next important factor, scenario asymmetry, measured here as the available bandwidth ratio, also constrains the design of such a system. Still, as initial results demonstrate, all of these requirements can be met in some heterogeneous scenarios, resulting in gain over pure mSCTP schemes. Further work will comprise more complex mobility patterns to better reflect the nature of heterogeneous wireless networks.

\section{ACKNOWLEDGMENT}

This work was supported in part by CYCIT (Spanish National Science Council) under grant TEC2006-09109 at UPC, and by University Research Program of Cisco Systems, Inc.

\section{REFERENCES}

[1] R. Stewart, "RFC 4960, Stream Control Transmission Protocol (SCTP)," September 2007. [Online]. Available: http://www.ietf.org/rfc/rfc4960.txt

[2] R. Stewart, Q. Xie, M. Tuexen, S. Maruyama, and M. Kozuka, "RFC 5061, Stream Control Transmission Protocol (SCTP) Dynamic Address Reconfiguration," September 2007. [Online]. Available: http://www.ietf.org/rfc/rfc5061.txt

[3] J. Iyengar, P. Amer, and R. Stewart, "Concurrent multipath transfer using sctp multihoming over independent end-to-end paths," IEEE/ACM Transactions on Networking, vol. 14, no. 5, pp. 951-964, October 2006.

[4] S. J. Koh and Q. Xie, "Mobile SCTP (mSCTP) for IP handover support, IETF draft," October 2005. [Online]. Available: http://tools.ietf.org/id/draft-sjkoh-msctp-01.txt, draft expired

[5] M. Tuexen, R. Stewart, P. Lei, and E. Rescorla, "RFC 4895, Authenticated chunks for the Stream Control Transmission Protocol (SCTP)," August 2007. [Online]. Available: http://www.ietf.org/rfc/rfc4895.txt

[6] M. Riegel and M. Tuexen, "Mobile SCTP, IETF draft," November 2007. [Online]. Available: http://tools.ietf.org/id/draft-riegel-tuexenmobile-sctp-09.txt, work in progress

[7] L. Ma, F. Yu, V. Leung, and T. Randhawa, "A new method to support UMTS/WLAN vertical handover using SCTP," IEEE Wireless Cоттиnications, vol. 11, no. 4, pp. 44-51, August 2004.

[8] Ł. Budzisz, R. Ferrús, and F. Casadevall, "SCTP multihoming performance in dynamically changing channels with the influence of link-layer retransmissions," in The 64th IEEE Vehicular Technology Conference (VTC 2006-Fall), September 2006, pp. 2624-2628.

[9] M. Chang, M. Lee, and S. J. Koh, "Transport layer mobility support utilizing link signal strength information," IEICE Transactions on Communications, vol. E87-B, no. 9, pp. 2548-2556, September 2004.

[10] Ł. Budzisz, R. Ferrús, A. Brunstrom, R. Grinnemo, K.-J. Fracchia, G. Galante, and F. Casadevall, "Towards transport-layer mobility: Evolution of sctp multihoming," Computer Communications, vol. 31, no. 5, pp. 980-998, March 2008.

[11] M. Honda, Y. Nishida, J. Nakazawa, and H. Tokuda, "Performance enhancement of transport layer handover on single-homed mobile nodes," IEICE Transactions on Communications, vol. E90-B, no. 10, pp. 26832692, October 2007.

[12] P. Natarajan, J. Iyengar, P. Amer, and R. Stewart, "Concurrent multipath transfer using transport layer multihoming: Performance under network failures," in IEEE Military Communications Conference (MILCOM 2006), October 2006.

[13] "The network simulator ns-2." [Online]. Available: http://www.isi.edu/nsnam/ns/ 